\title{
Small meteor ablation model: Applying to Perseid observations
}

\author{
V. Efremov ${ }^{1,2}$, O. Popova ${ }^{1}$, D. Glazachev ${ }^{1}$, A. Margonis ${ }^{3}$, \\ J. Oberst ${ }^{3,4}$ and A. Kartashova ${ }^{5}$ \\ 1 Sadovsky Institute of Geosphere Dynamics RAS, \\ Moscow, Russia,(E-mail: efremov.vv@phystech.edu) \\ 2 Moscow Institute of Physics and Technology \\ Moscow, Russia \\ 3 Technische Universität Berlin, Institute of Geodesy and Geoinformation \\ Science \\ Berlin, Germany \\ ${ }^{4}$ German Aerospace Center (DLR), Institute of Planetary Research \\ Berlin, Germany \\ 5 Institute of Astronomy RAS \\ Moscow, Russia
}

Received: August 1, 2021; Accepted: November 28, 2021

\begin{abstract}
We study the process of meteoroid interaction with the Earth's atmosphere, in particular, the effect of ablation. An ablation model is used, where mass loss of a meteoroid is determined using the saturated vapor pressure of the assumed meteoroid's substance. An automated method is suggested, where we estimate the physical parameters of a meteoroid by comparing data from observations and models of known parameters. Model constraints and features of the models are discussed.
\end{abstract}

Key words: Meteors - meteoroids - ablation model - Perseid shower

\section{Introduction}

Meteor bodies, along with asteroids and comets, carry important information about our Solar system, as the material, of which they are composed, testifies to the composition of matter in the early stages of Solar system evolution. Most part of these bodies do not reach the Earth's surface and do not become meteorites. A meteoroid entering the atmosphere produces radiation, ionization and sound waves. Analysis of these effects along with the meteoroid dynamics allows one to obtain information on the properties of the meteoric body and meteoric matter. In fact, the atmosphere is a detector to look at the properties of meteor bodies. The study of meteors permits one to learn more about the properties of asteroids and comets as they are the parent bodies of meteoroids. 
Table 1. Locations of observational sites

\begin{tabular}{llll}
\hline \hline Site & \multicolumn{3}{c}{ Coordinates } \\
& Longitude $\left(^{\circ}\right)$ & Latitude $\left(^{\circ}\right)$ & Altitude $(\mathrm{m})$ \\
\hline Mainalon & 37.26 & 22.59 & 1600 \\
Parnon & 37.65 & 22.29 & 1400 \\
\hline \hline
\end{tabular}

This is more cost-effective than space missions, although does not completely replace them.

Most meteoric bodies do not reach the surface of the Earth, so their properties have to be determined by remote sensing techniques. The problem of accurately determining the mass and other properties of the meteoroids from observational data remains unresolved despite the long history of studying meteor events (Subasinghe et al., 2017). The details of the interaction of meteor particles with the atmosphere are poorly known, so all their characteristics are determined with large uncertainties.

To estimate parameters of meteoric bodies (mass, density, etc.) from observational data, various ablation models are used (Popova et al., 2019). Two different ablation models were applied to a number of meteors in Efremov et al. (2018), and the results showed a significant difference in parameter estimates. In this paper we apply specific ablation models to observations of the Perseids and determine the parameters of the meteoroids. The model uncertainties and constraints are discussed.

\section{Observations and model}

\subsection{SPOSH camera and meteor observations}

A series of observing campaigns were carried out during Perseids activity in the period 2010-2016 from southern Greece (Margonis et al., 2019).

SPOSH (Smart Panoramic Optical Sensor Head) cameras were used for observations (Fig.1). The SPOSH camera is a flexible and sensitive system designed for imaging meteors not only from Earth observations but also from space probes orbiting the Earth or other planets (Christou et al., 2012). The camera is equipped with a highly sensitive back-illuminated 1024x1024 CCD chip and has a custom made optical system of high light-gathering power with a wide field-of-view (FOV) of $120 \times 120^{\circ}$. The camera was designed to capture meteors of magnitudes as faint as $+6^{m}$ moving at angular speeds of $5^{\circ} / \mathrm{s}$ (Oberst et al., 2011).

Two SPOSH camera systems were deployed at two sites (Mainalon and Parnon) (Table 1), separated by a $51.5 \mathrm{~km}-\mathrm{long}$ baseline. The camera fieldsof-view from the two stations overlap due to the near all-sky coverage of the cameras. To determine the meteoroid speed, a rotating shutter consisting of 


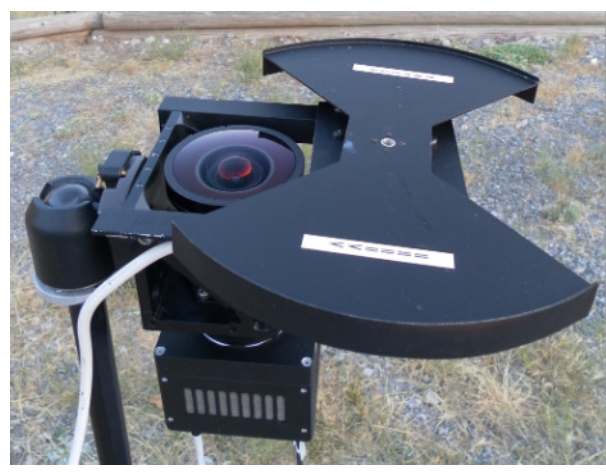

Figure 1. A SPOSH camera with a rotating shutter

two blades was mounted in front of the camera lens. The SPOSH data were acquired at Mt. Mainalon Station by Anastasios Margonis (TUB) and at Mt. Parnon Station by master students of the TUB along with DLR scientists. A software package developed for the SPOSH camera system was used (Margonis et al., 2018). The software consists of different modules, each carrying out distinct tasks in the characterization of a meteor event.

The range of absolute magnitudes of Perseid meteors recorded in August 2016 was from -6 to +2 magnitudes. Since the model used describes the ablation process of small meteor bodies (see below), meteors no brighter than $-2^{m}$ were chosen.

\subsection{Ablation model}

An ablation model is used to estimate parameters of meteor bodies (mass, density, etc.) from observational data. In this model the incoming energy flux is used for thermal radiation cooling, meteoroid heating and ablation (Lebedinets, 1980; Popova et al., 2019). The mass loss of a particle is determined taking into account the saturated vapour pressure (Knudsen-Lengmuir equation). To determine the parameters of meteoric bodies a selection of initial data is required, which will allow one to reconstruct the observations by solving a system of differential equations describing the change in height, velocity, mass and luminosity as a function of time.

The energy balance and mass loss equations are as follows:

$$
\begin{gathered}
\frac{1}{2} c_{h} \rho_{a} V^{3}=4 \varepsilon \sigma\left(T^{4}-T_{0}^{4}\right)-\frac{L}{\pi R^{2}} \frac{d M}{d t}+\frac{4}{3} R \rho c \frac{d T}{d t} \\
d M / d t=-4 \pi R^{2} P_{v}(T) \sqrt{\mu /(2 \pi k T)},
\end{gathered}
$$


where $M, V$ are mass and velocity of the body; $t$ is the time; $\rho_{a}$ is an atmospheric density at flight altitude; $g$ is the acceleration of gravity; $L$ is an ablation heat; $c_{h}$ is a heat transfer coefficient; $T$ is the body temperature; $T_{0}$ is an atmospheric temperature; $P_{v}$ is saturated vapor pressure; $\mu$ is average atomic mass of evaporating substance; $\rho$ is the meteoroid density; $R$ is the meteoroid radius; $\epsilon$ is an emissivity; $c$ is a heat capacity; $k$ is Boltzmann constant; $\sigma$ is Stefan-Boltzmann constant.

The energy conservation equation is supplemented by the equations of motion:

$$
\begin{gathered}
d V / d t=-c_{d} \pi R^{2} \rho_{a} V^{2} / M, \\
d \gamma / d t=-g \cos \gamma / V \\
d H / d t=-V \sin \gamma
\end{gathered}
$$

and by the equation for the light curve:

$$
I=-\tau \frac{d E_{k}}{d t}
$$

where $H$ is the height above the planet's surface; $\gamma$ is an entry angle (an angle of the trajectory inclination to the horizon); $c_{d}$ is the drag coefficient; $I$ is the intensity; $E_{k}$ is the meteoroid kinetic energy; $\tau$ is a luminous efficiency.

This model assumed that sublimation begins as soon as the meteoroid temperature starts to rise (Lebedinec \& Suskova, 1968; Love \& Brownlee, 1991; Moses, 1992; Campbell-Brown \& Koschny, 2004), and the temperature dependent mass loss rate being modelled using the KnudsenLangmuir formula (Bronshten, 1983).

The energy equation (1) is widely used in numerous papers devoted to the entry of small meteoroids in present form or with some modification (see review by Popova et al. (2019)). This ablation model is widespread in studies concerning the influx of micrometeorites, extraterrestrial matter, and cosmic dust as well the effects of this material on the atmosphere (see for example Plane (2012); Carrillo-Sánchez et al. (2016); Genge et al. (2017)).

For light curve fitting and meteoroid parameters determination the model is not used very often. The most elaborated model of this kind, which was applied to observational data, was suggested by (Campbell-Brown \& Koschny, 2004) to model Leonids meteors. Their dustball model includes equations (1-6) and suggests that meteoroids fragment into fundamental grains when the threshold temperature is reached and the volatile 'glue' is destroyed (thermal disruption). Kikwaya et al. (2011) used the thermal disruption model (Campbell-Brown \& Koschny, 2004) to fit observational data and to determine meteoroids parameters. It was required that the observed light curve and deceleration had to 
be fitted simultaneously by the modeled ones. Bulk densities of the meteoroids were determined as well as other characteristics were estimated (e.g. grain mass, threshold temperature)(Kikwaya et al., 2011). Later Campbell-Brown (2017) and Armitage \& Campbell-Brown (2020) modified the fragmentation mechanism of the thermal fracture model to better simulate meteor trails. Armitage \& Campbell-Brown (2020) investigated the ablation of two meteoroids. Their task was to determine whether high-resolution wakes provide enough data to constrain the mass distribution of small meteoroid grains.

Čapek \& Borovička (2017) used the ablation model (Eqs.(1-6)) as one of three possible ablation processes to describe the light curves and beginning heights of small iron meteoroids. They found that another process, i.e. the immediate removal of the liquid from the meteoroid's surface, is the most probable mechanism leading to the observed meteors (Čapek \& Borovička, 2017; Čapek et al., 2019).

The studies mentioned above combined the considered ablation model with fragmentation to fit observational data. Here the described ablation model without fragmentation is applied to Perseid meteor observations as a first approach to look on the model constraints in more detail.

\subsubsection{Model parameters}

The size and density of the meteoroid, determined from the observational data, depend not only on the fitted curves of luminosity and deceleration, but also on the assumptions about the luminous efficiency, heat transfer coefficient, expressions for the vapor pressure, etc., used in the ablation model. We will call these quantities the parameters of the model, although they may be dependent on both the properties of the meteoroid and the mode of its flight.

The luminous efficiency $\tau$ represents the fraction of a meteoroid's kinetic energy converted into light, either bolometrically,or in a specific spectral bandpass. In principle, the luminous efficiency depends on the chemical composition of the body and its velocity, size and height of flight. Subasinghe et al. (2017) collected estimates of different authors and demonstrated that the estimates differ from 10 to 100 times.

In the current application of the ablation model the luminous efficiency $(\tau)$ was supposed to be constant over the entire flight of the meteoroid and ranged from $1 \%$ to $5 \%$ (Weryk \& Brown, 2013; Subasinghe et al., 2017). In the most part of considered cases the luminous efficiency was fixed at $\tau=5 \%$. The power output by a zero magnitude meteor is one more factor of uncertainty. Weryk \& Brown (2013) estimated the power of $0^{m}$ meteor to be in the range $770-1610 \mathrm{~W}$ for a number of passbands assuming the temperature in the radiative area to be $4500-6000 \mathrm{~K}$. Here this value is fixed at $1100 \mathrm{~W}$ bolometrically.

The meteoroid was assumed to have a spherical shape. The heat transfer coefficient was supposed to be constant along the trajectory and to be equal to the value for the sphere in free-molecular mode $\left(c_{h}=1\right)$. The heat of ablation 
$(L)$ and the average atomic mass $(\mu)$ are determined by the substance and correspond to the used saturated vapour pressure $P_{v}$.

In the frame of this model meteoroid temperature is essential, and prewarming is important. The altitude $H \approx 300 \mathrm{~km}$ is a starting altitude, it is a reasonable compromise between accurate pre-heat accounting and calculation time. The numerical code is realized in WolframMath.

\subsubsection{Vapour pressure uncertainty}

In the ablation model under consideration, the mass loss is determined through the saturated vapour pressure of the meteoroid, for which different authors propose markedly different dependencies for the same substance (see below). The most commonly used pressure-temperature relationship is as follows:

$$
\log _{10} P_{v}=A-B / T
$$

where $A$ and $B$ are constants for a given substance and may vary for different temperature ranges. These constants are usually determined experimentally (Bronshten, 1983).

One of the main components of meteorites is silicates, including olivine ( $\mathrm{Ru}-$ bin, 1997). Olivine is a rock-forming mineral, a magnesia-iron silicate with the formula $(\mathrm{Mg}, \mathrm{Fe})_{2}\left[\mathrm{SiO}_{4}\right]$. Olivine forms a group or series of olivine. The $\mathrm{Fe}$ and $\mathrm{Mg}$ content varies between the two endmembers of the continuous isomorphic olivine series: forsterite (Fo) - $\mathrm{Mg}_{2}\left[\mathrm{SiO}_{4}\right]$ and fayalite $(\mathrm{Fa})-\mathrm{Fe}_{2}\left[\mathrm{SiO}_{4}\right]$. Olivine is one of the most common minerals on the Earth (Deer et al., 2013). In addition, olivine is one of the main minerals that make up extraterrestrial matter. Olivine can be found in of meteorites (Rubin, 1997), it is a major mineral in the lunar regolith (Arnold et al., 2016), and it is the most common mineral in samples brought back from the S-type asteroid Itokawa (Nakamura et al., 2011). The presence of olivine has also been confirmed in comet material (Hanner et al., 1997; Hanner, 1999) and in cosmic dust (Campins \& Ryan, 1989; Rietmeijer, 2004; Borovička et al., 2019). Olivine is present in particles belonging to comet $81 \mathrm{P} /$ Wild (Wild II) delivered by the Stardust mission (Zolensky et al., 2008). Rosetta mission confirmed the presence of olivine in $67 \mathrm{P} / \mathrm{Churyumov}$ Gerasimenko comet (Pätzold et al., 2019).

The saturated vapour pressure dependence on temperature for olivine and few related substances are shown on Fig. 2 according to a number of publications (references are given in Fig.2 caption). The melting temperature of olivine is in the range $1500-2100 \mathrm{~K}$ at atmospheric pressure in dependence on $\mathrm{Fe} / \mathrm{Mg}$ content (Costa et al., 2017). It can be seen that at a temperature of $2000 \mathrm{~K}$ the pressure has a spread of three orders of magnitude. Often dependencies are obtained experimentally in a narrow temperature range and extrapolation is used over a wider temperature range. Part of the scatter in the data may be associated with this. 


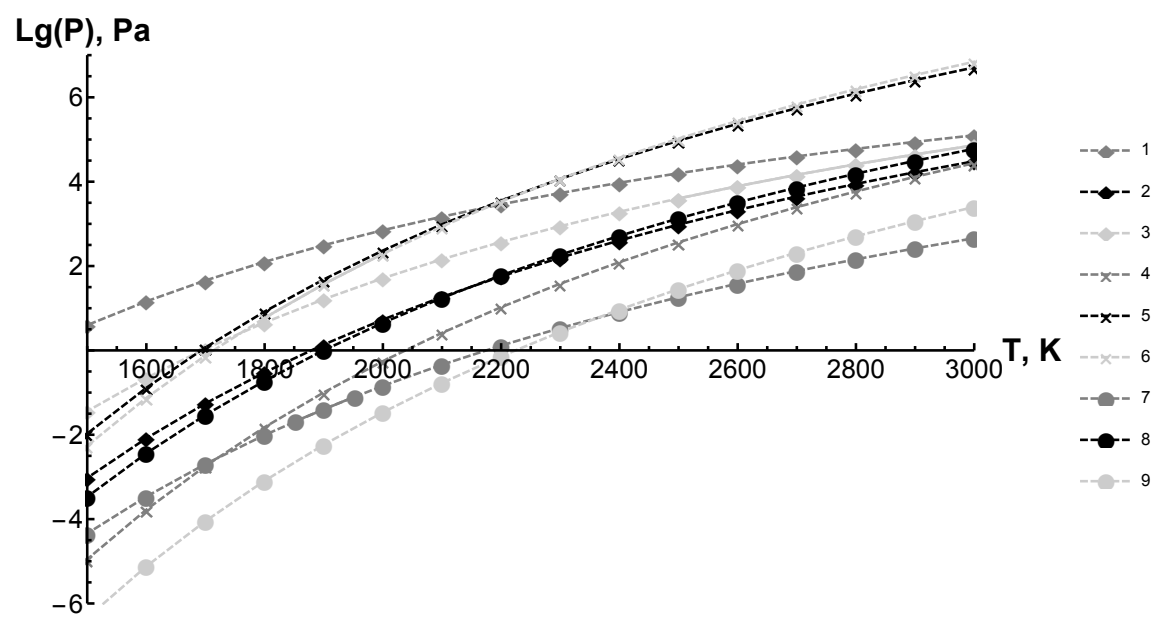

Figure 2. Dependence of saturated vapour pressure on temperature for olivine, silicates, iron and magnesium oxides according to different articles. The dashed lines mark the areas where the relationship for pressure is extrapolated. 1 - Baldwin \& Sheaffer (1971), olivine and chondrite, 2 - Costa et al. (2017), olivine, 3 - Costa et al. (2017), olivine, vapours Fe/Mg, 4 - Sekanina \& Chodas (2012), forsterite, 5 - Sekanina \& Chodas (2012), fayalite, 6 - Kazenas \& Chizhikov (1976), MgO, 7 - Kazenas \& Chizhikov (1976), FeO, 8 - Kimura et al. (1997), silicates, 9 - Kimura et al. (1997), quartz.

Campbell-Brown \& Koschny (2004) as well as subsequent publications (Kikwaya et al., 2011; Campbell-Brown, 2017; Armitage \& Campbell-Brown, 2020; Vida et al., 2020) estimated the dependence of the saturated vapor pressure for some meteor substance based on the solution of Clausius-Clapeyron equation and calibrated on atmospheric pressure. The resulting function would be located above the function for olivine (Fig. 2) and would lead to higher pressures at $1000-2000 \mathrm{~K}$.

Different studies used various dependences of saturated vapor pressure. For example, Moses (1992) studied the meteoroid influx and its effect in Neptune's atmosphere using the dependence close to Costa et al. (2017) data for olivine (Fig.2). Genge et al. (2017) modeled the entry heating of basaltic micrometeorites and used the ablation model with vapor pressure, for which the corresponding curve would be located slightly lower than quartz curve on Fig.2. Love \& Brownlee (1991) studied the micrometeoroids entry in the Earth's atmosphere assuming pressure dependence with higher values at $\mathrm{T} \approx 1000-2500 \mathrm{~K}$ than curves shown on Fig.2 but lower than the dependence by Campbell-Brown \& Koschny (2004). They claimed that the estimated rate of evaporative mass loss is consistent with that from the laboratory heating of Murchison chondrite. 
Noticeable number of publications, which consider the influx of chemical elements into the Earth's upper atmosphere, are using Chemical Ablation Model (CABMOD, Vondrak et al. (2008)). In CABMOD the vapour pressures of the species evaporating from the meteoroid are calculated using the MAGMA chemical equilibrium code. This model allows one to consider differential ablation and to predict the injection rates of individual elements. Here we will not consider the differential ablation and will apply different pressure dependences for olivine and related substances in the ablation model.

\subsubsection{The objective function}

The objective function is a measure of the difference between the simulated light curve and that of the meteor. It serves as the quantitative metric that represents the fitness of a given solution.

Few objective functions were tested to gain a better understanding of the limitations and strengths of different options. In the first type of function considered, the intensity values are used to construct the discrepancy function, which is minimized during the solution search. The first discrepancy $\Delta_{A I}$ is as following:

$$
\Delta_{A I}=\sqrt{\frac{\sum_{i}^{n}\left(I_{o}-I_{s}\right)^{2}}{n}},
$$

where $I_{o}$ is the observed intensity, $I_{s}$ is the solution intensity. The error is calculated at altitudes where observational data are available, $n$ is the number of observational points. The intensity varies by orders of magnitude during the meteor flight, so another function was constructed based on relative error $\Delta_{R I}$ :

$$
\Delta_{R I}=\sqrt{\frac{\sum_{i}^{n}\left(\left(I_{o}-I_{s}\right) / I_{o}\right)^{2}}{n}},
$$

Two other objective functions relied on meteor magnitude $\Delta_{A M}$ and relative magnitude $\Delta_{R M}$ :

$$
\begin{gathered}
\Delta_{A M}=\sqrt{\frac{\sum_{i}^{n}\left(M_{o}-M_{s}\right)^{2}}{n},} \\
\Delta_{R M}=\sqrt{\frac{\sum_{i}^{n}\left(\left(M_{o}-M_{s}\right) / M_{o}\right)^{2}}{n}},
\end{gathered}
$$

where $M_{o}$ is an observed magnitude, $M_{s}$ is a solution magnitude. In order to avoid $M_{0}$ values in the $(0, \pm 1)$ interval and corresponding effects, both light curves were artificially shifted by 10 magnitudes (parallel transfer).

The meteoroid parameter was determined from the light curve only, as the deceleration curve was not recorded accurately. The absence of significant deceleration (a loss of no more than $10 \%$ of the initial velocity) was controlled. It 
Table 2. Velocity, entry angle, absolute magnitude, and F-factor for the meteoroids in question. Estimates of mass from empirical relationships versus average mass obtained from four objective functions with saturated vapour pressure from Costa et al. (2017) (olivine, vapours $\mathrm{Fe} / \mathrm{Mg}$ ).

\begin{tabular}{|c|c|c|c|c|c|c|c|c|c|}
\hline \multirow[t]{3}{*}{$\overline{\text { meteor }}$} & \multirow{3}{*}{$\begin{array}{l}\mathrm{V} \\
\mathrm{km} / \mathrm{s}\end{array}$} & \multirow{3}{*}{$\begin{array}{l}\gamma \\
\left({ }^{\circ}\right)\end{array}$} & \multirow[t]{3}{*}{$\overline{M_{v}}$} & \multirow{2}{*}{\multicolumn{5}{|c|}{$\begin{array}{c}\text { Mass } \\
10^{-5} \mathrm{~kg}\end{array}$}} & \multirow[t]{3}{*}{$\mathrm{F}$} \\
\hline & & & & & & & & & \\
\hline & & & & Ja1967 & Ve1965 & Je2006 & Vi2018 & Modelling & \\
\hline$\overline{20160811 \_184336}$ & 60.7 & 12.8 & -1.14 & 14.27 & 39.35 & 110.90 & 11.30 & $1.73 \pm 0.17$ & 0.38 \\
\hline 20160811_221139 & 58.3 & 32.1 & -1.30 & 10.91 & 37.26 & 105.02 & 8.29 & .13 & 0.44 \\
\hline 20160811_200532 & 61.3 & 17.0 & -0.91 & 9.01 & 27.29 & 76.92 & 7.56 & 0.9 & 0.25 \\
\hline 20160811_202351 & 60.7 & 21.4 & -0.73 & 6.74 & 21.97 & 61.920 & 5.92 & $=0.08$ & 0.23 \\
\hline 20160 & 66.2 & 14.8 & -0.78 & 6.42 & 18.94 & 53.39 & 5.57 & 10 & 0.18 \\
\hline 20160811_205252 & 59.4 & 21.5 & -0.18 & 4.18 & 14.37 & 40.49 & 4.22 & .03 & 0.23 \\
\hline 20160811_190233 & 66.8 & 13.3 & 0.55 & 1.73 & 5.61 & 15.82 & 2.10 & 0.03 & 0.45 \\
\hline 20160811_205351 & 59.4 & 22.0 & -0.18 & 4.18 & 14.37 & 40.49 & 4.22 & 0.4 & 0.16 \\
\hline $2016 \mathrm{c}$ & 60.4 & 19.0 & -0.05 & 3.67 & 12.43 & 35.04 & 3.83 & 0.4 & 0.44 \\
\hline 20160811_205505 & 60.5 & 22.0 & 0.12 & 2.81 & 10.02 & 28.23 & 3.06 & $0.39 \pm 0.02$ & 0.31 \\
\hline 20160811_205716 & 63.5 & 24.0 & 0.64 & 1.30 & 4.97 & 14.00 & 1.62 & $0.15 \pm 0.05$ & 0.53 \\
\hline
\end{tabular}

Note: Ja1967: Jacchia et al. (1967); Ve1965: Verniani (1965); Je2006: Jenniskens (2006); Vi2018: Vida et al. (2018)

should also be noted that we did not account for fragmentation in our model, although its role may be notable (Subasinghe et al., 2017).

\section{Application of the ablation model to meteor data}

The ablation model described above was used to simulate 11 meteors, whose velocity, entry angle and maximal absolute magnitude are given in Table 2.

\subsection{Effect of the objective function}

Application of the ablation model described above to observational data allows one to determine meteoroid parameters (mass, size and density). Meteoroid size and density were chosen as independent variables in the search for a solution. A comparison of the simulated and observed light curves for meteor 20160811_184336 is shown in Fig. 3 obtained with saturated vapour pressure from Costa et al. (2017) (olivine, vapours $\mathrm{Fe} / \mathrm{Mg}$ ). Four objective functions were used. Various objective functions better describe different parts of the light curve. Solution obtained using the discrepancy $\Delta_{A I}$, based on the minimisation of the standard deviation, better describes the middle part of the light curve, other solutions better approximate the beginning and the end of the light curve.

Obtained parameters of meteor 20160811_184336 are collected in Table 3 for one saturated pressure dependence. The meteoroid mass estimate slightly 
Table 3. Meteor 20160811_184336 parameters. Vapor pressure $P_{v}$ from Costa et al. (2017)(olivine, vapours $\mathrm{Fe} / \mathrm{Mg}, L=6 * 10^{6} \mathrm{~J} / \mathrm{kg}$ ).

\begin{tabular}{lllll}
\hline \hline Error $\Delta$ & Mass, $10^{-5} \mathrm{~kg}$ & Density, $\mathrm{kg} / \mathrm{m}^{3}$ & Radius, $\mathrm{cm}$ & Value of $\Delta$ \\
\hline$\Delta_{A I}$ & 1.94 & 120 & 0.34 & $3.79 * 10^{-8}$ \\
$\Delta_{R I}$ & 1.50 & 226 & 0.25 & 0.36 \\
$\Delta_{A M}$ & 1.82 & 224 & 0.27 & 2.20 \\
$\Delta_{R M}$ & 1.65 & 204 & 0.27 & 0.46 \\
Mean value & $1.73 \pm 0.17$ & $194 \pm 43$ & $0.28 \pm 0.03$ & \\
\hline \hline
\end{tabular}

depends on the choice of the objective function (the scatter is about $15 \%$ or less), the radius also depends slightly on the residual function (the scatter does not exceed 20\%). At the same time, the density estimate demonstrates large scatter - up to two times.

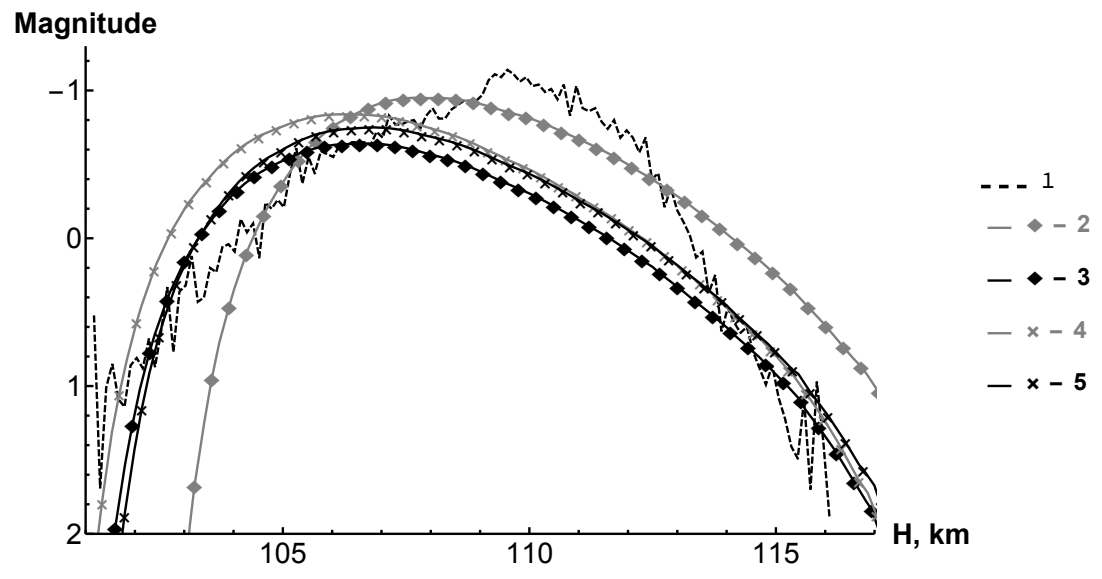

Figure 3. Light curve of meteor 20160811_184336 (dashed, curve 1) and model curves obtained with different objective functions: (2) $-\Delta_{A I}$, (3) $-\Delta_{R I}$, (4) $-\Delta_{A M}$, (5)$\Delta_{R M}$. Saturated vapour pressure from Costa et al. (2017)(olivine, vapours $\mathrm{Fe} / \mathrm{Mg}$ ). Corresponding meteoroid parameters are shown in Table 3.

However, the solution found does not always describe the light curve accurately. For example for meteor 20160811_200532 the solution using the saturated vapour pressure from Sekanina \& Chodas (2012) (fayalite) does not describe the light curve for objective functions $\Delta_{A M}$ and $\Delta_{R M}$ (Fig.4).This is due to the implemented solution search procedure. When the model solution has no data for brightness at certain altitudes (i.e. the model meteoroid burned out earlier than the observed one), a small value of magnitude $+10^{m}$ is formally assigned on 
Table 4. Parameters of meteor 20160811_200532, vapor pressure $P_{v}$ from Sekanina \& Chodas (2012)(fayalite, $L=2.46 * 10^{6} \mathrm{~J} / \mathrm{kg}$ ).

\begin{tabular}{lllll}
\hline \hline Error $\Delta$ & $\begin{array}{l}\text { Mass } \\
10^{-5} \mathrm{~kg}\end{array}$ & $\begin{array}{l}\text { Density } \\
\mathrm{kg} / \mathrm{m}^{3}\end{array}$ & $\begin{array}{l}\text { Radius } \\
\mathrm{cm}\end{array}$ & $\Delta$ \\
\hline$\Delta_{A I}$ & 0.92 & 611 & 0.15 & $3.60 * 10^{-8}$ \\
$\Delta_{R I}$ & 0.89 & 574 & 0.15 & 0.55 \\
$\Delta_{A M}$ & 4.97 & 585 & 0.27 & 2.90 \\
$\Delta_{R M}$ & 7.23 & 487 & 0.33 & 0.88 \\
Mean value & $3.50 \pm 2.72$ & $564 \pm 47$ & $0.23 \pm 0.08$ & \\
Mean value for $\Delta_{A I}$ and $\Delta_{R I}$ & $0.91 \pm 0.02$ & $593 \pm 19$ & 0.15 & \\
\hline \hline
\end{tabular}

Note: Uncertain values are marked gray

these altitudes. Under this condition, in some cases, the discrepancies $\Delta_{A M}$ and $\Delta_{R M}$ take the minimum values for the model light curves covering the entire height range, even if the model curves are located far from the observed ones. The continuation condition of the model curve will need to be reviewed and improved. At the moment unreliable solutions are not taken into account when estimating meteor parameters.

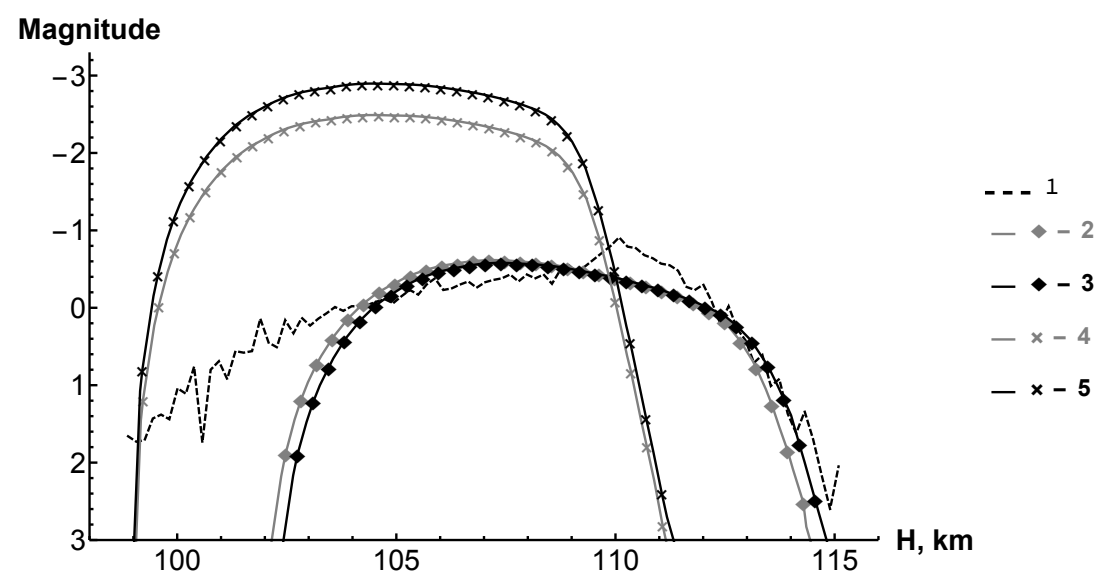

Figure 4. Meteor 20160811_200532 (dashed, curve 1) and model curves obtained with different objective functions: (2) - $\Delta_{A I},(3)-\Delta_{R I}$, (4) $-\Delta_{A M},(5)-\Delta_{R M}$. Saturated vapour pressure from Sekanina \& Chodas (2012)(fayalite). Corresponding meteoroid parameters are shown in Table 4.

The quality of the description of the light curves within the considered ablation model varies from meteor to meteor. Figure 5 shows the model and observed 
Table 5. Parameters of meteor 20160811_205716, vapor pressure $P_{v}$ from Sekanina \& Chodas (2012) (forsterite, $L=3.86 * 10^{6} \mathrm{~J} / \mathrm{kg}$ ).

\begin{tabular}{lllll}
\hline \hline Error $\Delta$ & Mass, $10^{-5} \mathrm{~kg}$ & Density, $\mathrm{kg} / \mathrm{m}^{3}$ & Radius, $\mathrm{cm}$ & Value of $\Delta$ \\
\hline$\Delta_{A I}$ & 0.15 & 485 & 0.09 & $4.37 * 10^{-8}$ \\
$\Delta_{R I}$ & 0.15 & 519 & 0.09 & 0.19 \\
$\Delta_{A M}$ & 0.15 & 512 & 0.09 & 0.21 \\
$\Delta_{R M}$ & 0.15 & 512 & 0.09 & 0.03 \\
Mean value & 0.15 & $507 \pm 3$ & 0.09 & \\
\hline \hline
\end{tabular}

light curves for meteor 20160811_205716. In this case, the parameters are the same for all the objective functions, and the density varies insignificantly (Fig.5, Table 5). The F-parameter (see below Section 3.3) is equal 0.5 (Table 2) for this meteor, one may assume that fragmentation is insignificant in this case.

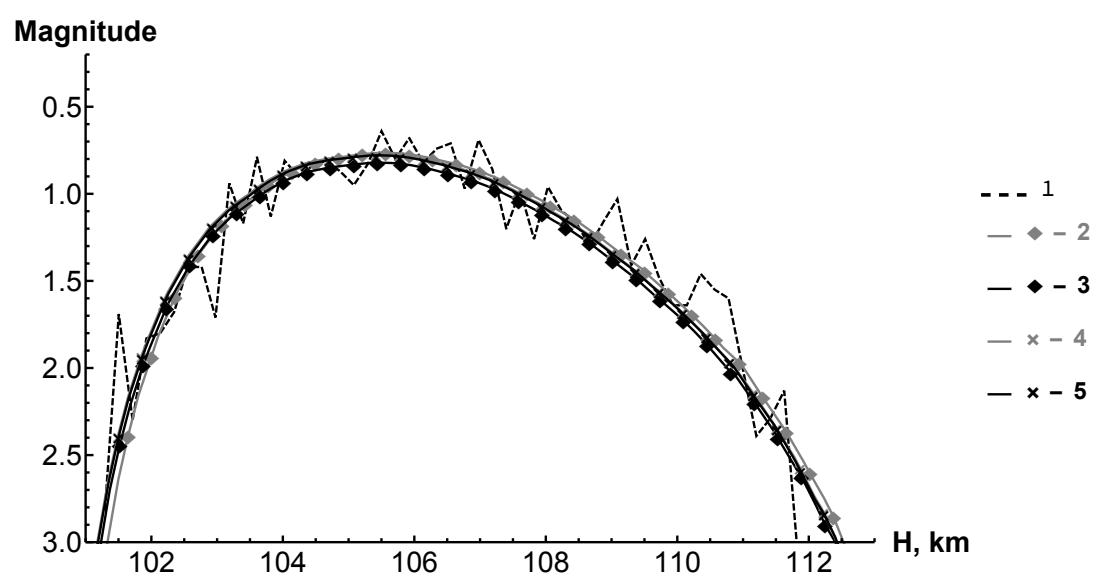

Figure 5. Meteor 20160811_205716 (dashed, curve 1) and model curves obtained with different objective functions: (2) $-\Delta_{A I},(3)-\Delta_{R I}$, (4) $-\Delta_{A M},(5)-\Delta_{R M}$. Saturated vapour pressure from Sekanina \& Chodas (2012) (forsterite). Corresponding meteoroid parameters are shown in Table 5.

\subsection{Influence of the dependence for saturated vapour pressure}

As mentioned above, the estimate of meteoroid parameters also depends on the saturated vapour pressure used. Figure 6 and Table 6 shows the parameter estimates for meteor 20160811_184336 using different pressures $P_{v}$ with the same objective function. Different dependences for vapor pressure significantly affect 
the shape of the light curve and the quality of its fit. At one of the pressures (quartz (Kimura et al., 1997)) the solution found does not fit the observational data. With a minimum of discrepancy, the meteoroid is not burned up (due to the high heat of ablation), but decelerated. No significant deceleration is recorded in the observational data, so this solution is not taken into account in the parameter estimation.

When comparing the estimates obtained at different pressures, it can be seen that the deviation from the average value of mass is not more than $10 \%$, and the size is not more than $35 \%$. In terms of density, the spread five times higher or more can five times higher or more.. Thus, it is possible to estimate the mass of the meteoroid quite accurately, but the density estimate appears uncertain.

Table 6. Parameters of meteor 20160811_184336 obtained with different pressure dependences $P_{v}$ and objective function $\Delta_{R I}$.

\begin{tabular}{llllll}
\hline \hline $\begin{array}{l}\text { Mass } \\
10^{-5} \mathrm{~kg}\end{array}$ & $\begin{array}{l}\text { Density } \\
\mathrm{kg} / \mathrm{m}^{3}\end{array}$ & $\begin{array}{l}\text { Radius } \\
\mathrm{cm}\end{array}$ & $\begin{array}{l}L \\
10^{6} \mathrm{~J} / \mathrm{kg}\end{array}$ & $\Delta$ & $P_{v}$ \\
\hline 1.50 & 226 & 0.25 & 6 & 0.36 & $\begin{array}{l}\text { C2019, olivine, } \\
\text { vapours Fe } / \mathrm{Mg}\end{array}$ \\
& & & & & C2019, olivine \\
1.67 & 119 & 0.32 & 6 & 0.42 & SCh2012, forsterite \\
1.63 & 77 & 0.37 & 3.86 & 0.59 & SCh2012, fayalite \\
1.74 & 516 & 0.20 & 2.46 & 0.45 & K1997, silicates \\
1.66 & 88 & 0.36 & 7.12 & 0.43 & Mean without quartz \\
$1.64 \pm 0.08$ & $205 \pm 164$ & $0.30 \pm 0.07$ & & $0.45 \pm 0.08$ & K1997, quartz \\
2.49 & 0.68 & 2.06 & 9.38 & 0.38 & \\
\hline
\end{tabular}

Note:C2019:Costa et al. (2017); SCh2012:Sekanina \& Chodas (2012);

K1997:Kimura et al. (1997). Uncertain values are marked gray.

\subsection{F-parameter}

As it was mentioned above, the fragmentation is not included in our model although it may critically affect the light curve (LC). For the coarse estimate of the fragmentation influence on the light curve, $F$-parameter was suggested, where $F$ is symmetry, a parameter with no units (Fleming et al., 1993). It is defined as the ratio of the one-sided width of the LC at $1^{m}$ below the peak, to the total width at this level. Formally $F$-parameter may be written as:

$$
F=\frac{t_{1}-t_{M}}{t_{1}-t_{2}},
$$

where $t_{1}$ is the time near the beginning of the meteor when its brightness is $1^{m}$ fainter than the peak, $t_{2}$ is the time near the meteor end when its brightness is $1^{m}$ below the peak, and $t_{M}$ is the time of the peak brightness (Brosch et al., 


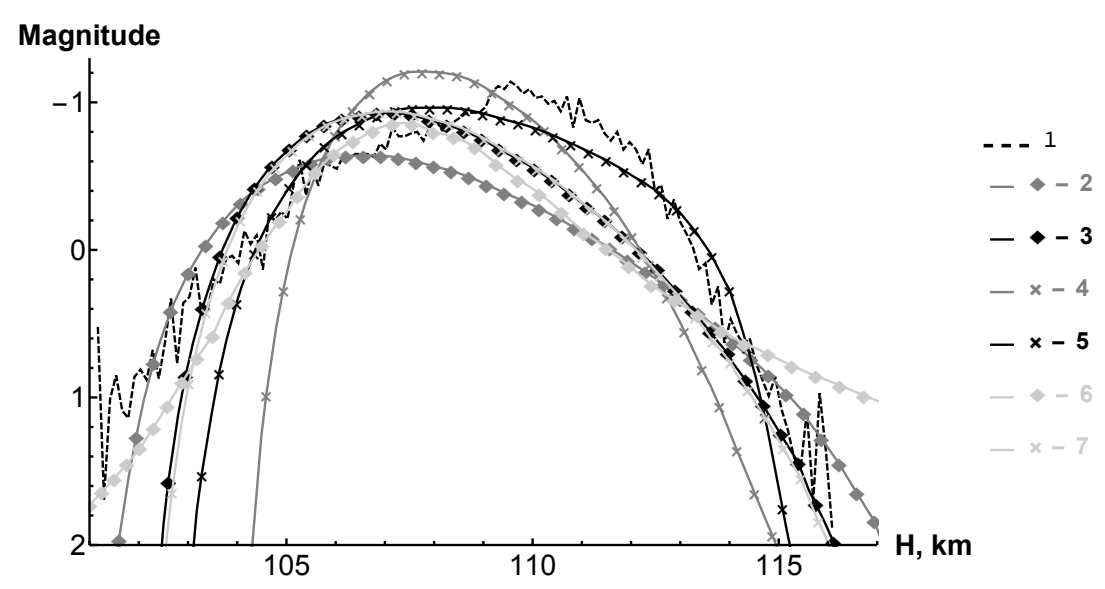

Figure 6. Luminosity curve of meteor 20160811_184336 (dashed, curve 1) and model curves obtained with $\Delta_{R I}$ discrepancy and using different dependencies for saturated vapour pressures: (2) - Costa et al. (2017) (olivine, Fe/Mg vapours), (3) - Costa et al. (2017) (olivine), (4) - Sekanina and Chodas (2012) (forsterite), (5) - Sekanina \& Chodas (2012) (fayalite), (6) - Kimura et al. (1997) (quartz), (7) - Kimura et al. (1997) (silicates). Corresponding meteoroid parameters are shown in Table 6.

2004). F-parameter is used to classify meteoric bodies by the shape of the light curve.

Observed light curves of meteors under consideration are characterized by $F \approx 0.16-0.53$, i.e. their maxima are in the middle or are shifted towards the beginning of the light curve (Table 2). Our data fall within the interval found in the paper by Koten et al. (2004), who determined that $F$ values for the Perseids are in the range $0.20-0.95$ with the maximum distribution at 0.54 . In the frame of our single body model $F$-parameter is about 0.5 -0.7 (Fig. 3-6), its value varies with applied pressure dependences.

The closer the observed $F$-parameter is to 0.50 , the more accurately its mass is determined. This model describes satisfactory meteoroids, whose $F$-parameter is about $0.4-0.5$. For these meteoroids the fragmentation effect is less significant, all four objective functions yield close parameters (Fig.5). The meteors with $F \approx 0.2-0.3$ are probably be better modeled with fragmentation included. The fragmentation model will be included into consideration in future studies.

There is no correlation between estimated meteoroid density and the $F$ parameter for our data sample. In our sample the size of the meteoroid has more influence than the shape of the light curve (see below). 


\subsection{Mass of the meteoroids}

Meteoroid mass is often determined based on empirical relations obtained during many years of observations and their interpretation. These relations used meteor maximum magnitude, velocity and entry angle (Jacchia et al. (1967); Verniani (1965); Jenniskens (2006); Vida et al. (2018)). Mass estimates based on these relationships are shown in the Table 2, along with simulation results for the meteors in question. Estimates collected in the Table 2 demonstrate large uncertainty in mass determination - more than an order of magnitude, which is long-standing problem of meteor research.

All considered meteoroids are Perseids, and their velocity are close to each other. The application of the ablation model is consistent with the well-known correlation between meteor brightness and meteoroid mass, the smaller is the maximum brightness, the smaller is the obtained mass of the meteoroid (Table 2, Fig.7).

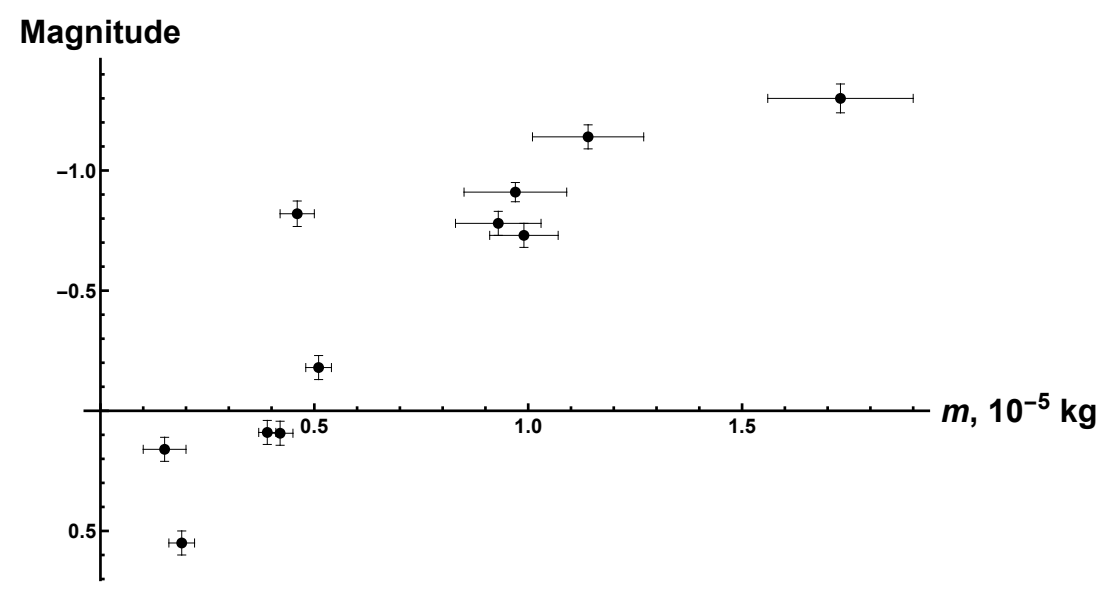

Figure 7. Relationship between meteor maximum brightness and meteoroid mass. Average mass value of the four objective functions with the saturated vapour pressure from Costa et al. (2017)(olivine, vapours $\mathrm{Fe} / \mathrm{Mg}$ ) was used.

The mass estimates we obtained are closest to the relationship from Vida et al. (2018) (difference by a factor of up to 10). Given that our estimates were obtained at a luminous efficiency of $5 \%$, perhaps at lower efficiency of $1 \%$ the mass estimates would agree with the relationship from Vida et al. (2018).

\subsection{Meteoroid density estimates}

The density of meteoroids in our model is determined with a large uncertainty. The scatter in density may reach several times for the same meteor using dif- 
ferent objection functions and different vapour pressures (see Table 3, 4, 5,6).

For the 11 meteors in question, the density spread is very wide, ranging from 50 to $1700 \mathrm{~kg} / \mathrm{m}^{3}$. The average density for all meteors is $\approx 362 \pm 237 \mathrm{~kg} / \mathrm{m}^{3}$, for individual meteors the spread of average densities is from $114 \pm 99$ to640 \pm $500 \mathrm{~kg} / \mathrm{m}^{3}$. In some solutions we get unusually low densities $\left(\approx 30-60 \mathrm{~kg} / \mathrm{m}^{3}\right)$. There is a weak correlation between density and meteor brightness. For fainter meteors the average density is higher than for brighter ones. The highest correlation occurs with saturated pressure for fayalite (Sekanina \& Chodas, 2012). There is no correlation between density and $F$-parameter. This is probably because meteoroids have different magnitudes, indicating a difference in size, and this in turn has a greater influence on the resulting density than the shape of the luminosity curve (which is characterised by the $F$-parameter).

Density estimation is influenced by both the choice of the objection function (variation up to 6 times) and the choice of dependence for pressure (up to 26 times). The highest particle densities are obtained using saturated vapour pressure for forsterite (Sekanina \& Chodas, 2012).

Our estimates are within the range of known cometary densities. Density of dust particles of comet $67 \mathrm{P} /$ Churyumov-Gerasimenko collected by the COSIMA spacecraft in the Rosetta mission ranges from 100 to $400 \mathrm{~kg} / \mathrm{m}^{3}$ (Hornung et al., 2016), the average density of the comet itself is estimated as $537 \mathrm{~kg} / \mathrm{m}^{3}$ (Pätzold et al., 2019). Results on the physical properties reveal that dust particles are essentially aggregates of grains, with high porosities, from at least $50 \%$ to much higher values (see review Borovička et al. (2019)).

A number of papers have derived estimates of Perseid meteor densities from observational data. Bellot Rubio et al. (2002) analyzed 413 photographic meteors with brightnesses from $-5^{m}$ to $+2.5^{m}$, obtained with Super-Schmidt cameras under single body and quasi-continuous fragmentation models. Both models assumed that all the energy of the incoming flux is spent on the ablation. The authors concluded that nearly three-quarters of the meteors in this set can be described within a single body model. The densities found under the singlebody model are always lower than in the frame fragmentation model. For the 5 Perseid meteors the density was estimated at $600 \pm 100 \mathrm{~kg} / \mathrm{m}^{3}$.

Babadzhanov (2003) estimated the densities of the 111 meteors observed in Dushanbe using the quasi continuous fragmentation model. For 44 Perseid meteors the density was estimated as $1300 \pm 200 \mathrm{~kg} / \mathrm{m}^{3}$. The mean mineral density of bright Perseid meteoroids (191 meteors of $-1^{m}$ to $-9^{m}$ magnitude) was estimated at $2250 \pm 40 \mathrm{~kg} / \mathrm{m}^{3}$ based on the solution of the heat-conduction equation (Babadzhanov \& Kokhirova, 2009). 35\% of these meteoroids have densities from 1000 to $2000 \mathrm{~kg} / \mathrm{m}^{3}$. In addition, a quasi-continuous fragmentation model has been used to simulate light curves and to estimate the meteoroid's bulk density and grain masses. This model allowed light curves to be reproduced and bulk densities to be estimated for the 97 Perseid meteors. The bulk density was estimated at $1200 \pm 200 \mathrm{~kg} / \mathrm{m}^{3}$ and the porosity was about $45 \%$ (Babadzhanov \& Kokhirova, 2009). 
Kikwaya et al. (2011) studied a large group of meteors with brightness from $+2.5^{m}$ to $+6^{m}$, fitting light and deceleration curves to meteoroid parameters. The investigation was based on the thermal disruption model (Campbell-Brown \& Koschny, 2004). The search for meteor body parameters was carried out in two stages. First the search was done over the entire parameter phase space and then over the selected area. For the 107 Perseid meteors the density of the meteoroids was in the range $420-820 \mathrm{~kg} / \mathrm{m}^{3}$.

Our density estimates turn out to be lower or comparable to those of other authors derived from the analysis of observational data with the help of different models. It is also worth bearing in mind that density estimates with fragmentation are typically higher.

\section{Conclusions}

A model describing the interaction of small meteoroids with the Earth's atmosphere is applied to observations of Perseid meteors in order to reproduce the light curves and to determine the parameters of meteoroids (density, size/mass). In this model, the mass loss of a meteoroid is determined using the saturated vapor pressure of the assumed meteoroid's substance. Dependencies of the saturated vapor pressure to the temperature of the same substance are different according to other authors, which affects the determination of the meteoroid parameters. An automated method to estimate the physical parameters of a meteoroid by comparing observational and model derived data with known parameters was suggested.

For testing the performance of the model optical meteor observations of SPOSH cameras in 2016 were used. The range of absolute magnitudes of meteors was selected as $-1.3^{m}-+1^{m}$. Parameters of meteoroids (density, size/mass) were obtained. The effects of the chosen dependence of saturated vapor pressure and used objective functions on meteoroid properties were considered.

The meteoroid parameters were determined from the light curves only, as the deceleration curves were not recorded, but the absence of significant deceleration (a loss of no more than $10 \%$ of the initial velocity) was controlled. It should also be noted that we have not considered fragmentation in our model, although its role may be significant.

The estimate of the mass of a meteoroid has little dependence on the choice of the objective function (deviation from the mean is no more than 15\%), and the radius also has a low dependence on the objective function (deviation from the mean no more than 20\%). At the same time, the dispersion in density is much higher.

Saturated vapour pressure dependences slightly affects the mass estimate (the deviation from the average value is no more than 10\%), more pronounced effect occurs for radius estimate (t deviation is no more than 35\%). Density spread may exceed five times. The vapour pressure dependence significantly 
affects the shape of the light curve, the quality of its fitting and the estimation of density.

The chosen model better describes meteoroids for which the $F$-parameter exceeds $\approx 0.4-0.5$. For meteoroids with a high $F$ value the effect of fragmentation is minimal, and the estimate of meteoroid parameters is only weakly dependent on the chosen objective function. For meteors with smaller $F$ values, it is likely that fragmentation should be taken into account. The fragmentation model will be included into consideration in future studies.

The density of meteoroids within our model is determined with a large uncertainty, which may reach several times for the same meteor using different objective functions and vapour pressures. The average density for all meteors is $\sim 362 \pm 237 \mathrm{~kg} / \mathrm{m}^{3}$. A comparison of our density estimates with data for cometary matter shows that our estimates are within the range of known cometary densities and appear to be lower or comparable to those of other authors, derived from the analysis of observational data in various models.

\section{Acknowledgements.}

AK to thank DAAD program "Research Stays for University Academics and Scientists, 2019"- 57440917. AM was financially supported by the German Research Foundation (DFG) - Project number (OB124/20-1).

\section{References}

Armitage, T. \& Campbell-Brown, M., Constraining the grain mass distribution of small meteoroids using high resolution data. 2020, Planetary Space Science, 186, 104915, DOI: $10.1016 /$ j.pss.2020.104915

Arnold, J. A., Glotch, T. D., Lucey, P. G., et al., Constraints on olivine-rich rock types on the Moon as observed by Diviner and M3: Implications for the formation of the lunar crust. 2016, Journal of Geophysical Research: Planets, 121, 1342, DOI: https://doi.org/10.1002/2015JE004874

Babadzhanov, P. B., Meteor showers associated with the near-Earth asteroid (2101) Adonis. 2003, Astronomy and Astrophysics, 397, 319, DOI: 10.1051/00046361:20021506

Babadzhanov, P. B. \& Kokhirova, G. I., Densities and porosities of meteoroids. 2009, Astronomy and Astrophysics, 495, 353, DOI: 10.1051/0004-6361:200810460

Baldwin, B. \& Sheaffer, Y., Ablation and breakup of large meteoroids during atmospheric entry. 1971, Journal of Geophysics Research, 76, 4653, DOI: 10.1029/JA076i019p04653

Bellot Rubio, L. R., Martínez González, M. J., Ruiz Herrera, L., et al., Modeling the photometric and dynamical behavior of Super-Schmidt meteors in the Earth's atmosphere. 2002, Astronomy and Astrophysics, 389, 680, DOI: 10.1051/00046361:20020672 
Borovička, J., Macke, R. J., Campbell-Brown, M. D., et al. 2019, Physical and Chemical Properties of Meteoroids, ed. G. O. Ryabova, D. J. Asher, \& M. J. CampbellBrown, 37

Bronshten, V. A. 1983, Physics of Meteoric Phenomena (Dordrecht, Holland, D. Reidel Publishing Company), 358

Brosch, N., Helled, R., Polishook, D., Almoznino, E., \& David, N., Meteor light curves: the relevant parameters. 2004, Monthly Notices of the RAS, 355, 111, DOI: 10.1111/j.1365-2966.2004.08300.x

Campbell-Brown, M., Modelling a short-wake meteor as a single or fragmenting body. 2017, Planetary Space Science, 143, 34, DOI: 10.1016/j.pss.2017.02.012

Campbell-Brown, M. D. \& Koschny, D., Model of the ablation of faint meteors. 2004, Astronomy and Astrophysics, 418, 751, DOI: 10.1051/0004-6361:20041001-1

Campins, H. \& Ryan, E. V., The Identification of Crystalline Olivine in Cometary Silicates. 1989, Astrophysical Journal, 341, 1059, DOI: 10.1086/167563

Carrillo-Sánchez, J. D., Nesvorný, D., Pokorný, P., Janches, D., \& Plane, J. M. C., Sources of cosmic dust in the Earth's atmosphere. 2016, Geophysics Research Letters, 43, 11,979, DOI: 10.1002/2016GL071697

Christou, A. A., Oberst, J., Elgner, S., et al., Orbital observations of meteors in the Martian atmosphere using the SPOSH camera. 2012, Planetary Space Science, 60, 229, DOI: $10.1016 /$ j.pss.2011.09.002

Costa, G. C. C., Jacobson, N. S., \& Fegley, Bruce, J., Vaporization and thermodynamics of forsterite-rich olivine and some implications for silicate atmospheres of hot rocky exoplanets. 2017, Icarus, 289, 42, DOI: 10.1016/j.icarus.2017.02.006

Deer, W., Howie, R., \& Zussman, J. 2013, An introduction of the rock-forming minerals (Mineralogical Society of Great Britain and Ireland, London, United Kingdom), 498

Efremov, V.V.and Popova, O., Glazachev, D., \& Kartashova, A., Determination of the meteor particles properties from observational data [in Russian]. 2018, Dynamic processes in geospheres, 150

Fleming, D. E. B., Hawkes, R. L., \& Jones, J., Light curves of faint television meteors. 1993, in Meteoroids and their Parent Bodies, ed. J. Stohl \& I. P. Williams, 261

Genge, M. J., Larsen, J., Van Ginneken, M., \& Suttle, M. D., An urban collection of modern-day large micrometeorites: Evidence for variations in the extraterrestrial dust flux through the Quaternary. 2017, Geology, 45, 119, DOI: 10.1130/G38352.1

Hanner, M. S., The Silicate Material in Comets. 1999, Space Science Reviews, 90, 99, DOI: $10.1023 / \mathrm{A}: 1005285711945$

Hanner, M. S., Gehrz, R. D., Harker, D. E., et al., Thermal Emission From The Dust Coma Of Comet Hale-Bopp And The Composition Of The Silicate Grains. 1997, Earth Moon and Planets, 79, 247, DOI: 10.1023/A:1006201820477

Hornung, K., Merouane, S., Hilchenbach, M., et al., A first assessment of the strength of cometary particles collected in-situ by the COSIMA instrument onboard ROSETTA. 2016, Planetary Space Science, 133, 63, DOI: 10.1016/j.pss.2016.07.003 
Jacchia, L., Verniani, F., \& Briggs, R. E., An Analysis of the Atmospheric Trajectories of 413 Precisely Reduced Photographic Meteors. 1967, Smithsonian Contributions to Astrophysics, 10, 1

Jenniskens, P. 2006, Meteor Showers and their Parent Comets

Kazenas, E. \& Chizhikov, D., The Pressure and Composition of the Vapor above Oxides of Chemical Elements [in Russian]. 1976

Kikwaya, J. B., Campbell-Brown, M., \& Brown, P. G., Bulk density of small meteoroids. 2011, Astronomy and Astrophysics, 530, A113, DOI: 10.1051/0004$6361 / 201116431$

Kimura, H., Ishimoto, H., \& Mukai, T., A study on solar dust ring formation based on fractal dust models. 1997, Astronomy and Astrophysics, 326, 263

Koten, P., Borovička, J., Spurný, P., Betlem, H., \& Evans, S., Atmospheric trajectories and light curves of shower meteors. 2004, Astronomy and Astrophysics, 428, 683, DOI: 10.1051/0004-6361:20041485

Lebedinec, V. N. \& Suskova, V. B., Evaporation and Deceleration of Small Meteoroids. in , Physics and Dynamics of Meteors, ed. L. Kresak \& P. M. Millman, Vol. 33, 193

Lebedinets, V. N. 1980, Dust in the upper atmosphere and cosmic space.

Love, S. G. \& Brownlee, D. E., Heating and thermal transformation of micrometeoroids entering the Earth's atmosphere. 1991, Icarus, 89, 26, DOI: 10.1016/00191035(91)90085-8

Margonis, A., Christou, A., \& Oberst, J., Observations of meteors in the Earth's atmosphere: Reducing data from dedicated double-station wide-angle cameras. 2018, Astronomy and Astrophysics, 618, A99, DOI: 10.1051/0004-6361/201832927

Margonis, A., Christou, A., \& Oberst, J., Characterisation of the Perseid meteoroid stream through SPOSH observations between 2010-2016. 2019, Astronomy and Astrophysics, 626, A25, DOI: 10.1051/0004-6361/201834867

Moses, J. I., Meteoroid ablation in Neptune's atmosphere. 1992, Icarus, 99, 368, DOI: 10.1016/0019-1035(92)90153-X

Nakamura, T., Noguchi, T., Tanaka, M., et al., Itokawa Dust Particles: A Direct Link Between S-Type Asteroids and Ordinary Chondrites. 2011, Science, 333, 1113, DOI: 10.1126/science. 1207758

Oberst, J., Flohrer, J., Elgner, S., et al., The Smart Panoramicfleming1993light Optical Sensor Head (SPOSH) - A camera for observations of transient luminous events on planetary night sides. 2011, Planetary Space Science, 59, 1, DOI: 10.1016/j.pss.2010.09.016

Pätzold, M., Andert, T. P., Hahn, M., et al., The Nucleus of comet 67P/ChuryumovGerasimenko - Part I: The global view - nucleus mass, mass-loss, porosity, and implications. 2019, Monthly Notices of the RAS, 483, 2337, DOI: 10.1093/mnras/sty3171

Plane, J. M. C., Cosmic dust in the earth's atmosphere. 2012, Chemical Society Reviews, 41, 6507, DOI: 10.1039/c2cs35132c 
Popova, O., Borovička, J., \& Campbell-Brown, M. D. 2019, Modelling the Entry of Meteoroids, ed. G. O. Ryabova, D. J. Asher, \& M. J. Campbell-Brown, 9

Rietmeijer, F. J. M., Dynamic pyrometamorphism during atmospheric entry of large ( 10 micron) pyrrhotite fragments from cluster IDPs. 2004, Met. Planet. Sci., 39, 1869, DOI: $10.1111 /$ j.1945-5100.2004.tb00082.x

Rubin, A. E., Mineralogy of meteorite groups. 1997, Met. Planet. Sci., 32, 231, DOI: 10.1111/j.1945-5100.1997.tb01262.x

Sekanina, Z. \& Chodas, P. W., Comet C/2011 W3 (Lovejoy): Orbit Determination, Outbursts, Disintegration of Nucleus, Dust-tail Morphology, and Relationship to New Cluster of Bright Sungrazers. 2012, Astrophysical Journal, 757, 127, DOI: 10.1088/0004-637X/757/2/127

Subasinghe, D., Campbell-Brown, M., \& Stokan, E., Luminous efficiency estimates of meteors -I. Uncertainty analysis. 2017, Planetary Space Science, 143, 71, DOI 10.1016/j.pss.2016.12.009

Čapek, D. \& Borovička, J., Ablation of small iron meteoroids-First results. 2017, Planetary Space Science, 143, 159, DOI: 10.1016/j.pss.2017.03.004

Čapek, D., Koten, P., Borovička, J., et al., Small iron meteoroids. Observation and modeling of meteor light curves. 2019, Astronomy and Astrophysics, 625, A106, DOI: $10.1051 / 0004-6361 / 201935203$

Verniani, F., On the Luminous Efficiency of Meteors. 1965, Smithsonian Contributions to Astrophysics, 8, 141

Vida, D., Brown, P., \& Campbell-Brown, M., Physical properties and radiant distribution of the Orionids as observed by the Canadian Automated Meteor Observatory's mirror tracking system. 2020, in European Planetary Science Congress, EPSC2020 456

Vida, D., Brown, P. G., \& Campbell-Brown, M., Modelling the measurement accuracy of pre-atmosphere velocities of meteoroids. 2018, Monthly Notices of the RAS, $\mathbf{4 7 9}$ 4307, DOI: 10.1093/mnras/sty1841

Vondrak, T., Plane, J. M. C., Broadley, S., \& Janches, D., A chemical model of meteoric ablation. 2008, Atmospheric Chemistry \& Physics, 8, 7015, DOI: 10.5194/acp8-7015-2008

Weryk, R. J. \& Brown, P. G., Simultaneous radar and video meteors-II: Photometry and ionisation. 2013, Planetary Space Science, 81, 32, DOI: 10.1016/j.pss.2013.03.012

Zolensky, M., Nakamura-Messenger, K., Rietmeijer, F., et al., Comparing Wild 2 particles to chondrites and IDPs. 2008, Met. Planet. Sci., 43, 261, DOI: 10.1111/j.19455100.2008.tb00621.x 